\title{
Das Nicht-Verstehen als Geste der Apologie der Bildmacht
}

Das Publikum stellte Fragen. Die Kritiker formulierten den Anspruch, die neuen, großformatigen Bilder zu verstehen, und konstatierten "befuddlement ${ }^{01}{ }^{11} \mathrm{Die}$ Institution des Museums sah die Aufgabe zu vermitteln, zu erklären. Die Künstler sollten antworten. Fragebogen wurden ihnen vorgelegt, die an Direktheit nichts zu wünschen übrig ließen.

"Was a specific model or scene used? Has the subject any special personal, topical or symbolic significance? « Doch dem bürokratischen Verhör wurden Negationen und absolut gesetzte Definitionen entgegengehalten: "Art as Art. Art from Art« oder "meaningless, contentless, formless, timeless « ${ }^{02}$ Der Aufforderung zur verbalen Erklärung stand eine zeitweise hilflose, zeitweise aggressive Verweigerung gegenüber.

Es ist die Erfolgsgeschichte des Abstrakten Expressionismus im Amerika der späten vierziger, fünfziger und frühen sechziger Jahre, die von einer komplexen Reflexion über den Anspruch des Verstehens von Bildern erzählt. Paradigmatisch kann das Hadern mit einem Verstehensbegriff nachvollzogen werden, in dem die Unmittelbarkeit des Kunstwerkes in seiner visuellen Manifestation gegen die sprachliche Erklärung gestellt wird. Eine Nachzeichnung ausgewählter Momente dieser Auseinandersetzung vermag auf die Konsequenzen verweisen, die dieser künstlerische Diskurs für die Selbstdefinition der kunsthistorischen Disziplin hatte. Sie verdichtete die Negationen und versuchte, ihnen als Betrachteranweisungen zu folgen. Nicholas Serota hat beschrieben, welche Mittel das MoMA in der Installation der Säle einsetzte, um das Museum zu einem Kapellenraum zu erhöhen.$^{03}$ Wie auf den Aufnahmen von Thomas

01 _ Edward Alden Jewell, »Modern Painters Open Show Today«, in: New York Times (2. Juni 1943), S. 28.

02 - 1963 antwortete Ad Reinhardt auf den Fragebogen des Museum of Modern Art mit diesen stilisierten Sätzen.

03 - Nicholas Serota, Experience or Interpretation: The Dilemma of Museums of Modern Art (Walter Neurath Memorial Lecture, 28), New York: Thames \& Hudson, 1996, S. 9: 》The walls are illuminated by a wash of artificial light dramatizing the paintings and seperating them 
Struth 1994 dokumentiert und thematisiert, leuchten die Ikonen der Abstrakten Expressionisten auratisch in der Stille. ${ }^{04}$

Auf der anderen Seite spann sie den Diskurs der Künstler weiter, um auf der Ebene des Kommentars den Status der visuellen Sprache des Bildes zu verteidigen.

\section{ERKLÄREN, VERSTEHEN UND ERLEBEN}

In den Diskussionen und der Theoriebildung der Abstrakten Expressionisten findet sich die Frage nach dem Verstehen als negative Folie, vor der die Frage nach einer "Bilderfahrung" in den Vordergrund tritt. Es wurde um einen Werkbegriff gerungen, der dem Status des Kunstwerkes als etwas Fremdes und Eigenständiges gerecht würde. Die Annäherung an diese Entität durch eine analytische Begrifflichkeit erschien als ikonoklastischer Akt. Im Namen einer »Questioning Public $\aleph^{05}$ wurden die Künstler in eine Verbalisierung getrieben, die im eklatanten Widerspruch stand zu einer ihrer zentralen Positionen: Sie versuchten, das Nichtverstehen als Potenz der Wirkung und Rezeption zu etablieren.

Immer wieder finden sich Äußerungen, wie diejenigen des Malers William Baziotes, die gegen die Qualität Verständlichkeit gerichtet waren. Die Erklärung erscheint als Moment der Vereinnahmung und Verfügbarmachung: »I have a horror of being easily understood ${ }^{06}$

Bei fast allen Künstlern des Abstrakten Expressionismus ist in den vierziger und fünfziger Jahren eine intensive Reflexion über die Gefahr der Entwertung der Bildwahrnehmung durch den Gestus der Erklärung festzustellen. Die Suche nach der Identifikation des Sujets, das Beharren auf der Frage nach der Intention, die Forderungen nach verbalen Erklärungen und Legitimationen, wurden als Auswüchse dieser Sprachkultur empfunden.

»At least at this time I have nothing to say in words which I would stand for. I am heartily ashamed of the things I have written in the past. This self-statement business has become a fad this season, and I cannot see myself just spreading myself with a bunch of statements everywhere, I do not wish to make. ${ }^{07}$

from the space of the spectator I...]. The control of space and light and the focus on a group of related paintings serve to intensify the experience of Pollock's work, creating that hushed transcendental mood which we associate with a chapel.«

04 - Vgl. hier auch die neue Studie zur Geschichte der Hängung im MoMA von Mary Anne Staniszewski, The Power of Display: A History of Exhibition Installations at the Museum of Modern Art, Cambridge, MA / London: MIT, 1998, und für die moderne Reflexion zur Problematik des ästhetischen Schutzraumes: Rudolf Bumiller / Caramelle Ernst / Chabrillart François u. a., Das Bild der Ausstellung (Katalog der Ausstellung Heiligenkreuzerhof Wien 1993), Wien: Hochschule für Angewandte Kunst, I993.

05 —The Questioning Public«; Titel des bulletin of the museum of modern art $15 / \mathrm{I}$ (Herbst I947).

06 - William Baziotes, "The Artist and His Mirror «, in: Right Angle 3/2 (Juni I949), S. 33.

07 Mark Rothko in einem Brief an Barnett Newman, 1950 datiert, Barnett Newman 
Zahlreich sind die Formulierungen, die anstelle der Aktivität des »Lesens der Werke« den Moment der »Begegnung« mit dem Werk neu zu bestimmen suchen. Das Bild sollte nicht auf etwas verweisen, sondern in seiner Präsenz unmittelbar eine eigene Realität erwirken. Den Reaktionen der Betrachter, den Wahrnehmungen der Interaktion zwischen ihnen und dem Werk kommt in diesen Entwürfen eine zentrale Stellung zu. Dieses Modell, das in einer intensiven Auseinandersetzung mit dem Status von Kultobjekten entwickelt wurde, zielte auf ein Wechselspiel zwischen der ehrfurchtsvollen Distanz zu einer fremden Welt und direkter Eingebundenheit in die Bildwirkung als existentieller aufrüttelnder Erfahrung.

»No possible set of notes can explain our paintings. Their explanation must come out of a consummated experience between picture and onlooker. ${ }^{08}$

Von Barnett Newman und Robert Motherwell wurden diese Gedanken zu einer Selbstdefinition der amerikanischen Kunst weitergetrieben, die sich radikal vom europäischen Bildverständnis abzusetzen suchte: »We are freeing ourselves of the impediments of memory, association, nostalgia, legend, myth, or what have you, that have been the devices of Western European painting. $\ll^{09}$

Als Gegenmodell nahm die amerikanische Avantgarde die Kategorie des Sublimen, durch neue Definitionen belebt, für sich in Anspruch. Die Kontemplation des Individuums vor dem einzelnen Werk sollte zu transzendentalen Erfahrungen führen. Der aus der Romantik stammende Topos der Moderne, das Schweigen vor dem Werk als Betrachteranweisung ${ }^{10}$, entfaltet seine Macht.

Es entstand eine Konstellation, in der der Anspruch auf ein Verstehen, das Verlangen

Papers, Archives of American Art, Roll 348 I.

08 - Zitat aus einem Statement verfasst von Adolph Gottlieb und

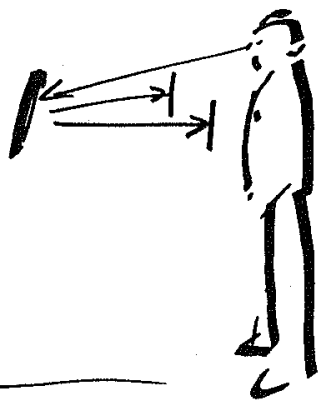
Mark Rothko mit Hilfe von Barnett Newman, abgedruckt bei Edward Alden Jewell, »Globalism Pops into View«, in: New York Times (I3. Juni 1943), S. 9; vgl. auch Bonnie Clearwater, "Shared Myths: Reconsideration of Rothko's and Gottlieb's Letter to the New York Times«, in: Archives of American Art Journal 24/I (1984), S. 23-25.

09 — $\gg$ The Ides of Art: 6 Opinions on What Is Sublime in Art? «, in: The Tiger's Eye 6 (Dezember 1948), S. 46-56.

10 - Wolfgang Kemp, »Zeitgenössische Kunst und ihre Betrachter: Positionen und Positionszuschreibungen«, in: ders. (Hg.), Zeitgenössische Kunst und ihre Betrachter (= Jahresring. Jahrbuch für moderne Kunst, Band 43), Köln: Oktagon, x996, S. 13-43; Wolfgang Kemp, »Die Kunst des Schweigens«, in: Thomas Koebner (Hg.), Laokoon und kein Ende: der Wettstreit der Künste, München: edition text + kritik, 1989, S. 96-1 18; Shimon Sandbank, »Poetic Speech and The Silence of Art«, in: Comparative Literature 46/3 (Sommer 1994), S. 225-239. 
nach Deutung, die begriffliche Leistung des Diskurses und das »buchstäbliche « Werk in ein ausgesprochen spannungsreiches Verhältnis traten. Sollte das Werk doch auf nichts mehr verweisen und seine Qualität durch die Identität von Sein und Bedeutung beweisen, so blieb die Instanz Sinnproduktion umstritten. ${ }^{11}$

\section{BEDEUTUNGSPRODUKTION}

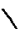

Die Selbstreferentialität und Geschlossenheit der Kunst ersetzt nämlich keineswegs die Bedeutungsproduktion im sprachlichen Diskurs. Vielmehr stand die Intention der Künstler gegen die Erkenntnis, dass es die Betrachtenden sind, die den Akt der Sinnproduktion zu übernehmen hätten. Die Geste des Verstehens durfte die Idee der »Offenheit«, der potentiellen Bedeutungsvielfalt, nicht zerstören, die spezifische Selbstreflexion des Mediums nicht gefährden. Wie konnte von ihnen also Sprache eingesetzt werden, die als Rezeptionsanweisung die Bilder vor einem »falschen« Diskurs schützen würde?

Ein zentrales Indiz für diese Reflexion findet sich im Umgang mit den Bildtiteln. ${ }^{12}$ In der berühmten Diskussion »Artists Sessions at Studio 35«, die der Maler Robert Goodnough edierte ${ }^{13}$, diskutierten etwa Hedda Sterne, Adolph Gottlieb, Ad Reinhardt, Robert Motherwell und Willem de Kooning kontrovers über das Dilemma einer Betitelung, die vom Publikum erwartet würde. Als Alternativen wurden abstrakte Nummerierungen ebenso erwogen wie poetische Allusionen oder deskriptive, klassifizierende Bezeichnungen wie "Landschaft« oder »Portrait«. Sehr schnell führte die Diskussion zum Wunsch, ein Bildverständnis zu bewahren, welches das Bild in seiner unmittelbaren Realität nicht durch Zeichenfunktionen beschneiden würde. »The question is how to name what yet has been unnamed « fasste Motherwell zusammen. Die Behauptung, wie sie der Maler Jimmy Ernst in die Diskussion einführte, dass ihn die Titel nicht störten, weil sie nichts mit dem Bild zu tun hätten, steht im Widerspruch zu den sensiblen Reaktionen auf den Umgang der Museen und Galerien mit der Titelgebung der Werke. Clyfford Still reagierte empört über falsche Betitelungen ebenso wie Newman sich um falsche und richtige Namensgebungen seiner Werke stritt. ${ }^{14}$

Mehrfach drehen sich die Argumente um den zentralen Punkt, nämlich die Beziehung zwischen Titel und Rezeptionsverhalten. Wird mit der Bezeichnung eines Sujets dem Werk etwas hinzugefügt, dass das Bild selbst nicht leiste?

11 -Vgl. die Diskussion des Problems bei Rainer Metzger, Buchstäblichkeit: Bild und Kunst in der Moderne, Köln: König, 2004, S. ז48-157.

12 - Es gibt bisher wenig Untersuchungen zur Problematik von Bildtiteln. Vgl. aber Felix Thürlemann, Kandinsky über Kandinsky: Der Künstler als Interpret eigener Werke, Bern: Benteli, 1986; John C. Welchman, Invisible Colors: $A$ Visual History of Titles, New Haven / London: Yale University Press, 1997.

13 __ Robert Goodnough, »Artists Sessions at Studio 35 (1950)«, in: Modern Artists in America (April 1950), S. 8-22, hier besonders S. 15.

14 _-Vgl. Peter J. Schneemann, Who's Afraid of the Word, Freiburg i. Brsg.: Rombach, 1998. 
Wird nahegelegt, dass das Bild auf etwas anderes im Sinne eines Symbols oder gar einer Metapher verweise? Immer wieder steht dagegen die Verteidigung des in sich selbst geschlossenen Werkes: Ibram Lassaw: A work of art $»$ is « like a work of nature.

Das Problem der Bildtitel steht in direktem Zusammenhang zu den mehrschichtigen und häufig kontradiktorischen Interessen an einer fruchtbaren Wechselbeziehung zwischen Betrachtung, Bedeutungsproduktion und Dichtkunst. ${ }^{15}$ Es wurde nach einem Diskurs gesucht, der eine Alternative darstellen könnte zur vermittelnden Sprache der konservativen amerikanischen Kunstkritik, die auf Identifikation und Benennung und Distanz ausgerichtet war. Es ging um eine Sprache, die ebenso als Rezeptionsanweisung dienen könne, wie eine Lösung des Anspruches des Verstehens anzuzeigen vermöge. Man mag diese Experimente als Reaktion auf die konservative Kritik von Emily Genauer, Howard Devree ${ }^{16}$ oder John Canaday deuten.

Die Abstrakten Expressionisten sahen in der dichterischen Sprache ihrer Freunde Modelle für eine Dimension des Wortes, die ihren Vorstellung der Offenheit und des Prozesshaften gemäß sein würde. Der Sprache des Verstehens wurde eine Sprache gegenübergestellt, die in poetischer Form eine assoziative Begleitung erprobte. ${ }^{17}$

Der Kunstkritiker Thomas Hess regte die Literaten an, sich in der von ihm herausgegebenen führenden Kunstzeitschrift Art News als Kritiker zu betätigen, so wie er Künstler einlud, über die Arbeit von Kollegen zu schreiben. Es entstanden Texte, die den Schlüsselbegriff der Abstrakten Expressionisten, »experience«, in Formen persönlich geprägter Ekphrasis umsetzten. Selbst eine Kunsthistorikerin und Kritikerin wie Dore Ashton versuchte, in Aphorismen Bilderfahrungen einzufangen und Verstehen zu vermitteln.

In vulgarisierter Form fanden diese Experimente Eingang in Ausstellungskataloge der Museen und $\mathrm{Ga}$ lerien. Es etablierte sich ein Modell für Ausstellungspublikationen, das biographische Daten des Künstlers, sein Foto-

15 - Vgl. zur allgemeinen Problematik: Stefan Greif, Die Malerei kann ein sehr beredtes Schweigen haben: Beschreibungskunst und

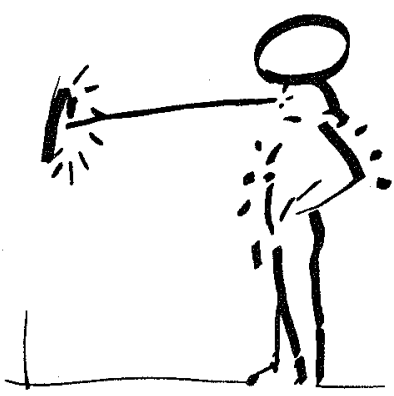
Bildästhetik der Dichter, München: Fink, 1998; Anne-Kathrin Reulecke, Geschriebene Bilder: Zum Kunst- und Mediendiskurs in der Gegenwartsliteratur, München: Fink, 2002; Helmut Pfotenhauer / Gottfried Boehrn: Beschreibungskunst - Kunstbeschreibung: Ekphrasis von der Antike bis zur Gegenwart, München: Fink, 1995 .

16 - Howard Devree, »Modern Babel: A Study of Confusion in the Art World«, typescript, 1947. Washington D.C.: Archives of American Art.

17 - Vgl. etwa den Dichter Frank 0'Hara; dazu: Marjorie Perloff, Frank O'Hara: Poet Among Painters (1977), Chicago / London: University of Chicago Press, 1998. 
portrait und ein häufig graphisch gestaltetes poetisches Statement neben der Reproduktion seines Werkes zu einem festen Gefüge, mit wechselseitigen Implikationen verband. Nicht selten verkam die Kommentarsprache dabei zur leeren theatralischen Geste.

Komplexer waren dagegen die Experimente, die Maler, Dichter, Philosophen und Kritiker in den sogenannten »little Magazines« umsetzten. So erschien etwa die Suche nach einer Wiederbelebung der Begrifflichkeit des Sublimen nicht zufällig in der Zeitschrift The Tiger's Eye. Diese kleine Zeitschrift (19471949) verband Reproduktionen der zeitgenössischen amerikanischen und europäischen Malerei mit Gedichten, philosophischen und literarischen Essays. Ähnliche versuche wurden von den kurzlebigen Periodika »Iconograph« (1940-1947), »Possibilities« (1947/48), »Instead« (I948) und »It is« (I9581965) unternommen. ${ }^{18}$

Immer wieder fanden hier Versuche ihren Platz, den Status von Bild und Text in der Disposition der Vermittlung zu klären. Die Diskussionen um rezeptionsästhetische Modelle Spiegelten sich unmittelbar in der Gestaltung der Zeitschriften wider. Wie ist im Prozess des Verstehens das Verhältnis zwischen selbstbezüglichem, reinem Kunstwerk und Intentionalität? Obwohl in The Tiger's Eye Texte und Werkreproduktionen parallel erschienen, sollte die Eigenständigkeit beider erhalten bleiben.

Die Statements zur Konzeption der Zeitschrift selbst, wie etwa im Oktober Heft von 1947 abgedruckt, beziehen sich auf die Implikationen dieser Parallelität und deuten ein Verständnis der Künstleräußerung an, das für The Tiger's Eye von eklatanter Bedeutung ist. Die Herausgeber stellen klar, dass alle veröffentlichten Texte als »Literatur« und nicht als Ergänzung der Bilder zu betrachten seien.

Das Statement beginnt mit den Worten: »That a work of art, being a phenomenon of vision, is primarily within itself evident and complete. ${ }^{19}$ Dieses Statement verweist ebenso auf die Diskussionen der Literaturszene wie die Brechung in der Zuordnung von Werk und Autor. Die Beiträge, Bilder oder Texte, können nur über das in der Mitte der jeweiligen Ausgabe versteckte Inhaltsverzeichnis einem Autor zugeordnet werden. Die Angabe des Autors ist vom Werk bewusst getrennt.

Die Betonung auf das Eigenleben des Werkes in Unabhängigkeit vom seinem Schöpfer, das Beharren auf die Unübersetzbarkeit der Medien wurde parallel in dem berühmten Aufsatz von Monroe C. Beardsley und W. K. Wimsatt, ,The Intentional Fallacy<, diskutiert, der in $»$ The Sewanee Review erschien. $^{20}$

Die Frage der Übersetzbarkeit einer künstlerischen Manifestation, die unmittelbar an ein Medium gebunden sei, griff eine Haltung auf, wie wir sie

18 - Ann Eden Gïbson, Issues in Abstract Expressionism: The Artist-run Periodicals, Ann Arbor MI: UMI Research Press, r990.

19 - $\gg$ The Tiger's Eye has the following convictions that will guide its publication of art«, The Tiger's Eye I (1947), S. 76.

$20-$ Monroe C. Beardsley / W. K. Wimsatt, »The Intentional Fallacy«, in: The Sewanee Review 54 (1946), S. $3^{-23}$. 
aus der Romantik kennen. Bereits die Galeriegespräche der Romantiker kreisten um diese Problematik. Die »Anschauung « findet sich als unübersetzbares Moment beschrieben, das nur unter der Trauer eines Verlustes in eine Verbalisierung überführt werden könne. Die Potenz des Schrittes zur Sprache als einem analytischen Instrument muss im Willen zum Verstehen ständig befragt werden. Dagegen steht jedoch die Suche nach dem Einsatz der Sprache als Kommunikationsmedium. Im Rahmen eines Gespräches kommt ihr die Funktion zu, Deutungen als Bedeutungen entstehen zu lassen.

Das Verstehen setzt hier keinen abgeschlossenen Sinn voraus. Das Moment des Nichtverstehens, die Annäherung an ein als Nichtverstandenes gesetztes, generiert Verstehen und Bedeutung im Sinne eines hervorbringenden, kreativen Aktes. Es lässt sich nicht mehr unterscheiden, welche Wirkung vom Bild und welche vom Betrachter ausgeht:

»Waller [Dichter]: Das trockene Urtheilen wollen wir gern den Kunstverständigen überlassen. Allein wir werden doch das Recht haben, Eindrücke mitzutheilen, die unser eignes Werke sind?

Reinhold [Maler]: Eignes Werk? wie so? sie wären also willkührlich?

Waller: Selbstthätigkeit ist noch wesentlich von Willkühr unterschieden. Eine Wirksamkeit kann nach der gegebenen Anregung nothwendig und doch unser eigen seyn. Daraus, dass die Eindrücke eines Kunstwerkes bey verschiednen Personen an Reichthum und Tiefe und Zartheit so erstaunlich weit von einander abstehen, leuchtet es ein, wie viel auf das ankommt, was der Betrachter mit hinzubringt. $\ll^{21}$

Noch deutlicher führte dieses Modell einer modernen Rezeptionsästhetik Clemens Brentano in seinem Kommentar zu Caspar David Friedrich aus:

»- und das, was ich in dem Bilde selbst finden sollte, fand ich erst zwischen mir und dem Bilde, nämlich einen Anspruch, den mir das Bild tat, indem es denselben nicht erfüllte. $\aleph^{22}$

Seine Beobachtungen führt er über in die Form des Dialoges

21 August Wilhelm, Carolin und Friedrich Schlegel und Fichte, "Die Gemälde: Ein Gespräch«, in: Athenaeum, Band II, I. Stück, Berlin: Heinrich Fröhlich, r799, S. 47.

22 -Clemens Brentano, „Verschiedene Empfindungen vor einer Seelandschaft von. Friedrich, wor-

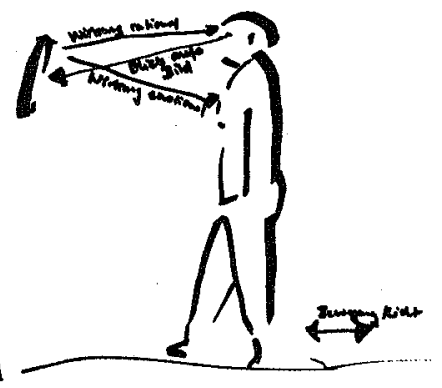
auf ein Kapuziner (Bei einer Kunstausstellung 18 ro)«, in: Friedmar Apel (Hg.), Romantische Kunstlehre, Erankfurt am Main: Deutscher Klassiker Verlag, 1992, S. 35 I-356. 
als angemessene Form. Hier scheinen viele Konzepte der modernen Rezeptionsästhetik vorweggenommen zu sein. ${ }^{23}$

\section{RÜCKWIRKUNGEN AUF DAS SELBSTVERSTÄNDNIS DER KUNSTGESCHICHTE}

Das Interesse an solchen Konstruktionen der aktiven Kunstbetrachtung und des produktiven Nichtverstehens schützte die Künstler jedoch nicht vor der schmerzhaften Erfahrung eines zerstörerischen Missverstehens. Die Reaktionen auf die ersten Einzelausstellungen von Newman, Rothko oder Still verdeutlichen das ungelöste Problem, das im Verhältnis zwischen dem Anspruch auf eine unmittelbare Bildmacht und einem Diskurs der konditionierenden Anweisung liegt. Beim Glauben an die eigenständige Dimension des Bildes blieb die Angst vor dem Wort des Betrachters bestehen. Es klingt immer wieder die Sorge durch, dass eine Verweigerung der Rezeptionsanweisung das Werk zu zerstören imstande sei. ${ }^{24}$

Clyfford Still brachte erbittert die verbalen Reaktionen auf seine Bilder auf einer Tafel vor dem Eingang zu seiner Ausstellung von 1947 an. Als Konsequenz, die er aus den Missverständnissen zog, kündigte er an, nicht mehr auszustellen. $^{25}$

Auch Mark Rothko gehörte neben Still und Newman zu den Künstlern, die ausgesprochen verletzt auf Reaktionen von Betrachtern reagierten, die sich der Anweisung entzogen. I947 publizierte The Tiger's Eye Rothkos Statement eines Märtyrers, der seine Leiden als die seiner Werke beschreibt:

"A picture lives by companionship, expanding and quickening in the eyes of the sensitive observer. It dies by the same token. It is therefore a risky act to send it out into the world. How often it must be impaired by the eyes of the unfeeling and the cruelty of the impotent who would extend their affliction universally. $\ll^{26}$

$23-$ Gerhard Kurz, "Vor einem Bild: Zu Clemens Brentanos sverschiedenen Empfindungen vor einer Seelandschaft von Friedrich, worauf ein Kapuziner«, in: Jahrbuch des freien deutschen Hochstifts (1988), S. I 28-r44.

24 - Die ikonoklastischen Anschläge auf die Bilder Newmans finden immer wieder das Interesse der Kunstgeschichte. Sie werden als Beweis für die Existenz einer intensiven Bildwirkung eingesetzt. Bereits in der ersten Ausstellung muss ein Bild beschmiert worden sein (The Art Digest [15. März 1950], S. 5). - Dieser Vorfall blieb in den Studien zu den späteren Anschlägen auf Gemälde Newmans unberücksichtigt. - 1982 wurde ein Anschlag auf das Berliner Bild »Who's Afraid of Red, Yellow and Blue IV« (1969-1970) verübt; 1986 war »Who's Afraid of Red, Yellow and Blue III«(1967-1968) Ziel eines Anschlages; der gleiche Täter schlug I989 nochmals in Antwerpen zu. Vgl. zuletzt Dario Gamboni, The Destruction of Art: Iconoclasm and Vandalism since the French Revolution, London: Reaktion Books, I997, S. 207-21I.

25 — Justus Jonas-Edel, Clyfford Stills Bild vom Selbst und vom Absoluten: Studien zur Intention und Entwicklung seiner Malerei (Dissertation), Köln: König, 1995, Kap. II, Anm. 70. 26 - Mark Rothko, "Statement«, in: The Tiger's Eye I/2 (1947), S. 44, wiederabgedruckt im Ausstellungskatalog I5 Americans, New York: Museum of Modern Art, 1952, S. 21; vgl. zu 
Im Falle Newmans und seiner frühen Ausstellungen stellte die Kritik den Anspruch der Unmittelbarkeit den philosophierenden Künstler entgegen. Die kurze, abwertende Kritik eines der wichtigsten Historiographen des Abstrakten Expressionismus enthält einen versteckten Hinweis auf diesen Umstand. ${ }^{27}$ Hess betitelt den Künstler einerseits als »one of Greenwich Village's best known homespun ${ }^{28}$ aestheticians $\ll$, der die "products of his meditations« zeigen würde. Andererseits beschreibt er Newmans Werke als optische Experimente. Damit sprach die Kritik die doppelte Apologie des Diskurses von Newman an, der das von ihm und seinen Freunden verkündete Ideal einer »sprachlosen Kunst« selbst zerstören musste.

Als visuelle Experimente beschrieben, sah Newman seine Farbflächen in die Nähe einer zunehmenden Akzeptanz der neuen Kunst als Dekoration gerückt und auch durch formalistische Interpretationen ihrer Kraft beraubt. Nach zwei erfolglosen Ausstellungen verzichte Newman lange Zeit auf eine Einzelausstellung.

In der Apologie des Bildes als eines unmittelbaren Ausdrucks, der als ungebrochene eigene Wahrheit physisch erfahrbar sei, entstanden verschiedene verbale Kommentarformen. In ihrer Rhetorik und ihren Figuren wirkten sie entscheidend auf den kunsthistorischen Umgang besonders mit Werken der Abstraktion zurück. Zwei Ebenen und zwei unterschiedliche Funktionen der Sprache im Verstehensprozess werden dabei miteinander vermengt.

Auf der einen Seite findet sich der Versuch, die spezifische Gesetzmäßigkeit des Mediums Bild und dessen Wahrnehmung parallel in eine poetisch geformte Sprache zu überführen und damit den Verstehensprozess im Fluss zu halten. Dem Kunstwerk wird dabei eine grundsätzliche Hermetik zugesprochen. ${ }^{29}$ Diese Konstellation des Nicht-

Rothko das neue Werkverzeichnis, David Anfam, Mark Rothko: The Works on Canvas - Catalogue Raisonné, New Haven: Yale University Press; Washington, D.C.: National Gallery of Art, 1998.

27 - Thomas B. Hess, "Review《, in: Art News 49/1 (1950), S. 48. Hess

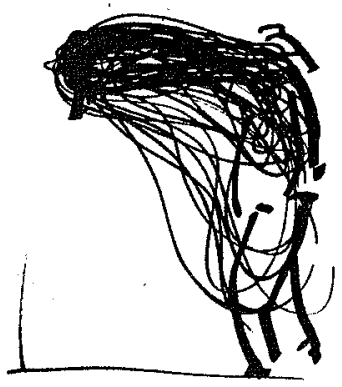
schließt mit einer Wendung, in der die anderen Künstler und nicht die normalen Galeriebesucher als eigentliche Adressaten Newmans angesprochen werden, im Sinne einer Aggression: »Newman is out to shock, but he is not out to shock the bourgeoisie - that has been done. He likes to shock other artists. « Eine der wenigen positiven Reaktionen stammte signifikanterweise von einer Kritikerin, der er seine Bilder lange erläutert hatte: Aline Louchheim, »By Extreme Modernists«, in: New York Times (4. Januar 1950), Sec. 2, S. 9. Ihre Kritik wurde in The Art Digest ( 5 . Februar I950), S. 5, unter dem Titel »Too Many Words « des hohlen Jargons bezichtigt. Die Polemik Peyton Boswells wurde von Newman scharf erwidert, The Art Digest (I5. März I950), S. 5 .

$28-$ homespun $=$ selbstgesponnen.

29 _ Vgl. dazu die grundlegende Arbeit von Ulli Seegers, Alchemie des Sehens - Hermetische Kunst im 2o. Jahrhundert: Antonin Artaud, Yves Klein, Sigmar Polke, Köln: König, 2003. 
verstehens wird gestaltet und beschworen - nicht jedoch hermeneutisch, deutend aufgehoben. In einem Diskurs um die mediale Differenz wird dem Bild und seiner Anschauung eine Eigendynamik zugeschrieben, die die Potenz zu einem »anderen « Verstehen in sich berge.

Auf der anderen Seite entsteht ein umfangreicher Meta-Diskurs im Sinne eines Kommentars ${ }^{30}$, der ein Begriffsgerüst und eine theoretische Legitimation der Verteidigung des Bildes als Sonderform des Verstehens bereitstellt. Der Umgang mit diesem Diskurs, mit Schlüsselbegriffen und Rezeptionsanweisungen, stellt für die Kunstgeschichte ein ungelöstes Problem dar. ${ }^{31}$

In der Rezeptionsgeschichte und der Historiographie des Abstrakten Expressionismus hat der Umgang mit dem inhaltlichen Anspruch, der mit der Utopie einer Bildmacht verknüpft war, immer wieder zu Kontroversen geführt. $198_{5}$ berief sich Michael Bockemühl ebenfalls auf Newman, um die manschauende Tätigkeit der Rezeption als eigentliche Bildproduktion« zu thematisieren. ${ }^{32}$ Newman dient als Beispiel für einen Maler, der erkannt habe, dass kein Gehalt außerhalb des Bildes bestehe. Der Eigenwert des Bildes als Objekt stehe gegen den Darstellungswert. Newman leiste die ultimative Aufhebung jeder Dichotomie zwischen Form und Inhalt. In Newmans Werken sei kein Sinn zu finden, sondern dieser müsse durch die Betrachtung selbst erzeugt werden. ${ }^{33}$

Gerne werden in diesem Kontext nochmals die Aussagen der Künstler zitiert, die auf die Verweigerung der Sprache verweisen und Interpretationen zurückweisen. Die Fiktion des "unschuldigen Betrachters« muss dazu dienen, eine Bildmacht zu bezeugen, die moralische und metaphysische Werte vermittle, ohne den Diskurs zu benötigen. Die Auflösung der Dichotomie von Form und Inhalt gelingt nur durch ein empathisches Befolgen sowohl der suggestiven Titel als auch der Anweisungen des theoretischen »Kon-Textes«.

Das Anzeigen des Anspruches ersetzt die direkte literarische Vorlage. Für die Rezeption liegt die Funktion des Diskurses in der begrifflichen Überhöhung der sinnlichen Erfahrung. Eine Rezeptionssteuerung durch die Einbindung in eine Installation, wie sie im Sinne der fotografischen Veranschaulichung Newmans denkbar wären, verfolgten die Abstrakten Expressionisten nicht weiter. Eine zu freie und aktive Rolle schrieben sie dem Betrachter in ihrem Wirkungsmodell zu.

Sehr viel respektloser und radikaler als die kunsthistorischen Annäherungen befragten Künstler ab den sechziger Jahren die Auseinandersetzung der Abstrakten Expressionisten um Bild und Kommentar und vor allem um Bild und Diskurs. Es ist eine zeitliche Verschiebung festzustellen zwischen künstlerischer und kunsthistorischer Reflexion über die mit der Beschwörung der

30 - Vgl. zu diesem Begriff Christian Bracht, Kunstkommentare der sechziger Jahre: Funktionen und Fundierungsprogramme, Weimar: VDG, 2002.

31 - W. J. T. Mitchell, ")Ut Pictura Theoriac: Abstract Painting and the Repression of Lan-

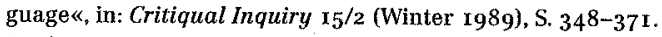

32 Michael Bockemühl, Die Wirklichkeit des Bildes, Stuttgart: Urachhaus, 1989, S. I5.

33 — Vgl. dazu Matthias Bunge, "Die Wirklichkeit des Bildes: Eine kritische Auseinandersetzung mit Michael Bockemühls These von der >Bildrezeption als Bildproduktion«, in: Zeitschrift für Ästhetik und Allgemeine Kunstwissenschaft 35 (I990), S. I3I-I89. 
Bildmacht einhergehenden Implikationen. Während die Kunstgeschichte die Rezeptionsanweisungen der Abstrakten Expressionisten affirmativ befolgte, setzte mit der konzeptuellen Kunst eine radikale Thematisierung der Utopien der fünfziger Jahre ein.

Als Beispiel für die Bearbeitung der beschriebenen Positionen sind etwa John Baldessaris Bilder zu nennen. Baldessari ließ in den späten sechziger und frühen siebziger Jahren Dogmen und Maxime des Diskurses um die Bildmacht von einem professionellen Schildermaler auf die Leinwand malen. Die Referenzen sind mehrschichtig. Er zieht sich, abgesehen von der Signatur, aus dem Prozess des Malens zurück, seine künstlerische Leistung liegt im Zitat. Bildtitel und gemalter Schriftzug werden eins. »A Two-Dimensional Surface Without Any Articulation is a Dead Experience ${ }^{34}$ von 1967 spielt mit Greenbergs Ideologie der Zweidimensionalität der Leinwand. Jonathan Borofsky stellte I983 den »Chattering Man« als Betrachter seinen abstrakten Bildern gegenüber, ${ }^{35}$ die durch endlose Ziffernfolgen bezeichnet sind. Seine Silhouette des Museumsbesuchers zerstört die Doktrin der Stille mit plärrenden Lautsprechern: »chatter, chatter, chatter«.

Das Verhältnis der Kunstwahrnehmung zum Versagen analytischer Sprache bildete eine entscheidende Grundlage für museumspädagogische Bemühungen, kunsthistorischer Theorieansätze und der Gestaltung von Ausstellungsräumen. Es ist zu beobachten, wie die Kunstgeschichte sich damit das Problem des künstlerischen Kommentars zu Eigen machte. ${ }^{36}$ Die Entwicklung der Disziplin im Sinne eines Weges von der Ekphrasis zur wissenschaftlichen Beschreibung ${ }^{37}$ scheint sich notwendiger Weise dabei häufig umzudrehen.

Die eindrücklichen Analysen der Werke der Abstrakten

34 - John Baldessari, "A Two-Dimensional Surface Without Any Articulation is a Dead Experiencer,

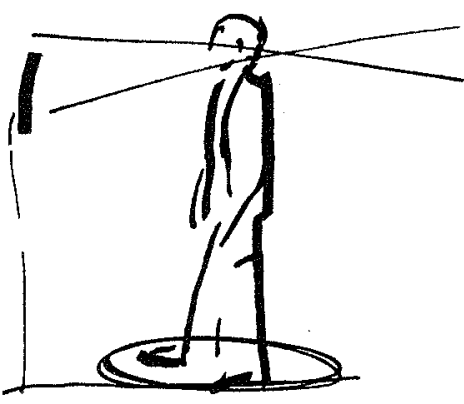
I967. Acryl auf Leinwand, $144.8 \mathrm{x}$

I77. $4 \mathrm{~cm}$, im Besitz des Künstlers; Mark Rosenthal, Critiques of Pure Abstraction: A Traveling Exhibition Organized and Circulated by Independent Curators Incorporated, New York: Independent Curators Incorporated, 1995, 0.S.; vgl. auch Modes of address: language in art since rg6o (Katalog der gleichnamigen Ausstellung), New York: Whitney Museum of American Art, 1988.

35 - Jonathan Borofsky, sUntitled at 2,835,666«, r983, Tinte und öl auf Papier, $203 \times$ $256.5 \mathrm{~cm}$ mit »Chattering Man«, Mixed media, $203 \times 58 \times 33 \mathrm{~cm}$, New York: Paula Cooper Gallery!

$36-V g l$. dazu Oskar Bätschmann, Bild-Diskurs: Die Schwierigkeit des Parler Peinture, Bern: Benteli, I977, S. 33-56.

37 - Raphael Rosenberg, "Von der Ekphrasis zur wissenschaftlichen Bildbeschreibung: Vasari, Agucchi, Félibien, Burckhardt«, in: Zeitschrift für Kunstgeschichte $5^{8 / 3}$ (1995), S. 2973 I 8 . 
Expressionisten durch die Kunstgeschichte bilden eine Ekphrasis, indem sie durch die Macht des Wortes, der Neologismen und des poetischen Ausdruckes dem Bild eine neue Wirkungspotenz verleihen. Die Tätigkeit der künstlerischen Betitelung findet sich in gespiegelter Form im Sprechen der Rezipienten über die abstrakten Bilder wieder.

Die Rhetorik der künstlerischen Verteidigung des Status der Bilder mit der Berufung auf deren Eigenmächtigkeit wurde und wird zum Anlass genommen, im geschützten Galerieraum Übungen des geduldigen Sehens durchzuführen. Der Kunstgeschichte diente die Verweigerung der Künstler in der eigenen Rechtfertigung, als Vermittler ästhetischer Erfahrung zu agieren. ${ }^{38}$

Deutlich wird diese Spiegelung in den Versuchen einer Verbalisierung der Bilderfahrung unter gleichzeitiger Reflexion eines notwendigen Scheiterns. ${ }^{39}$ Die Kunstgeschichte griff einen Lösungsansatz der Künstler selbst auf und führte deren Rhetorik fort. Suggestiv kann man die berühmten Fotografien, die den dramatisch gestikulierenden Newman als Vermittler seiner Kunst vor den Gemälden zeigen, neben die Aufnahme stellen, die Arnold Bode in seinem Vermittlungsengagement vor dem Gemälde Pollocks auf der zweiten documenta festhält.

Für Max Imdahl ${ }^{40}$ lag im Verhältnis zwischen Moderner Kunst und begrifflicher Leistung der Rezipienten ein Problem, das er in ausführlichsten Beschreibungen zu lösen suchte. ${ }^{41}$ Die Erfahrung der intensiven Bildbetrachtung setzte er in eine Sprache um, welche die Begegnung mit dem Kunstwerk auf eine ethische Ebene hebt.

Die Sprache der Künstler, die das Werk in seinem Status und seinem Anspruch verteidigten, findet sich hier im Engagement des Kunsthistorikers gespiegelt. Imdahl versuchte, mit einer möglichst präzisen Sprache zu einer reinen Bilderfahrung hinzuführen. Sein Einsatz für die »Seinsvalenz $\star^{42}$ des Kunstwerkes wurde jedoch von seinen Schülern gerne herangezogen, um das

$38-$ Als Beispiel für den Missbrauch dieser Rhetorik vgl. R. Bowman, "Words and Images: A Persistent Paradox «, in: Art Journal 45/4 (1985), S. 335-343.

39 __ Eva S. Sturm, Im Engpass der Worte: Sprechen über moderne und zeitgenössische Kunst, Berlin: Reimer, 1996; Uwe Wieczorek, „Über das Schweigen in der Kunst: Neuzeitliche Beispiele unter besonderer Berücksichtigung des 20: Jahrhunderts«, in: Festschrift für Hartmut Biermann, Weinheim: VCH, 1990, S. $25 \mathrm{I}-274$.

$40-$ Vgl. zu Imdahl auch Jürgen Stohr, »Ein Unverständnis in Bochum 1982? Das Kunstschaffen von Yves Klein und Gerhard Richter als Hinweis auf neuere Perspektiven einer Theorie ästhetischer Erfahrung«, in: Zeitschrift für Ästhetik und Allgemeine Kunstwissenschaft $39 / 1$ (I994), S. 9I-I 29.

41 - Max Imdahl hat in einer nicht weiter ausgeführten Bemerkung diese Möglichkeit angezeigt, dass "die Theorie als Anweisung zum Sehen an die frei gewordene Stelle der Ikonographie als einer Anweisung zum Verstehen tritt« (meine Hervorhebung), Max Imdahl, »Die Rolle der Farbe in der neueren französischen Malerei: Abstraktion und Konkretion «, in: Wolfgang Iser (Hg.), Immanente Ästhetik - Ästhetische Reflexion: Lyrik als Paradigma der Moderne, München 1966, S. I96; vgl. dazu Stefan Gronert, »Künstler-Theorie und Bildbegriff: Zu einigen Konsequenzen für das Verständnis moderner Malerei«, in: Zeitschrift für Ästhetik und allgemeine Kunstwissenschaft 34/1 (1989), S. $1_{3}^{1-143}$.

42 _ Der Begriff stammt von Hans-Georg Gadamer. 
Verhältnis der Moderne zur Sprache für eine grundsätzliche Mystifizierung des Aktes der Bildbeschreibung umzunutzen:

"Ihn [Imdahl] zog jener Ring des Schweigens nachdrücklich an, der das Kunstwerk eigentlich charakterisiert. Ein Schweigen, das zu uns in eigentümlicher Intensität zu sprechen vermag, und seinen besonderen Sinn unmittelbar mitteilt, den wir allerdings schwerlich einzuordnen vermögen in die bekannten historischen Deutungsmuster. Denn seine Sprache ist die der Sichtbarkeit, die wir durch die Tätigkeit unserer Wahrnehmung selbst organisieren, deren Übersetzung in die begrifflich bestimmte Sprache aber immer ein schwieriger, nur unter Verlusten möglicher Prozess ist. $\ll^{43}$

Die Kunstgeschichtsschreibung des Abstrakten Expressionismus griff die warnenden Aussagen der Künstler um die Dichotomie von Kunstwerk und analytischer Sprache auf, um vielleicht ein letztes Mal das Credo der Moderne zu verkünden, wie es in ihrer Meistererzählung formuliert wird, die Reinheit des visuellen Mediums in Einklang mit einer unübersetzbaren Botschaft, die nur im unschuldigen Erleben zu fassen sei. Gleichzeitig, ab der Mitte der sechziger Jahre, negierte bereits der Diskurs der Konzept Art radikal »modernism's fetish of visuality«. Thomas Crow hat herausgearbeitet, wie damit der Widerspruch zwischen der Artikulationsfähigkeit der Künstler und dem beanspruchten Status ihrer Werke als reine Visualität zu einem Ende fand. ${ }^{44}$

Ich behaupte jedoch, dass die Kunstgeschichtsschreibung der Abstraktion einen wesentlichen Grundstein legte für eine Bewegung innerhalb der Kunstgeschichte, die nach dem spezifischen Erkenntnispotential des Bildes und seiner Wahrnehmung fragt. In den Ansätzen des Iconic Turn ist die Frage nach der medialen Bedingtheit einer Kunstrezeption erneuert worden. Die Materialität und ihre Erfahrung werden unter Verstehensaspekten aufgewertet, die nicht nur die Vielschichtigkeit, das Uneindeutige betonen, sondern denen eine Qualität zugeschrieben wird, die jenseits der sprachlichen Artikulation unmittelbar in kognitive Prozesse einwirke.

In diesem Aufgreifen und Fortführen der künstlerischen Apologie des Bildes sollte die Kunstgeschichte jedoch darauf achten, nicht implizit normativ aufzutreten. Die Sinnlichkeit eines Kunstwerkes darf nicht als Notwendigkeit für Kunst postuliert werden. Denn bei aller »Medienkompetenz« darf nicht vergessen werden, dass sich zeitgenössische Kunst auch im diskursiven Raum selbstbewusst behauptet.

43 - Heínz Liesbrock, »Die Unersetzbarkeit des Bildes«, in: ders. Die Unersetzbarkeit des Bildes: Zur Erinnerung an Max Imdahl, Münster: Westfälischer Kunstverein, 1996, S. 8-1 8. 44 -Thomas Crow, "Unwritten Histories of Conceptual Art: Against Visual Culture«, in: Thomas Crow (Hg.), Modern Art in the Common Culture, New Haven / London: Yale University Press, 1996, S. $212-242$.

Alle Abbildungen aus dem Forschungsprojekt der HGKZ »Kunst und Qualität«, 2003. 